PROCEEDINGS OF THE

AMERICAN MATHEMATICAL SOCIETY

Volume 129, Number 12, Pages 3753-3755

S 0002-9939(01)06120-2

Article electronically published on June 27, 2001

\title{
SYSTEMS OF LINEAR ORDINARY DIFFERENTIAL EQUATIONS WITH BOUNDED COEFFICIENTS MAY HAVE VERY OSCILLATING SOLUTIONS
}

\author{
D. NOVIKOV
}

(Communicated by Carmen C. Chicone)

\begin{abstract}
An elementary example shows that the number of zeroes of a component of a solution of a system of linear ordinary differential equations cannot be estimated through the norm of coefficients of the system.
\end{abstract}

Bounds for oscillations. In 1 it was shown that a linear ordinary differential equation of order $n$, with real analytic coefficients bounded in a neighborhood of the interval $[-1,1]$, admits a uniform upper bound for the number of isolated zeros of a solution defined on this interval. The analyticity condition can be relaxed; only the boundedness of the coefficients matters. Probably, the simplest result in this spirit is the following theorem for the linear ordinary differential equation

$$
y^{(n)}(t)+a_{1}(t) y^{(n-1)}(t)+\cdots+a_{n}(t) y(t)=0
$$

with continuous coefficients on $[\alpha, \beta] \subset \mathbb{R}$.

Theorem 1 ([3, 4]). If the coefficients of the differential equation (1) are uniformly bounded by the constant $C \geq 1$ (that is, $\left.\max \left\{\left|a_{i}(t)\right|: i=1, \ldots, n\right\} \leq C\right)$, then a solution defined on $[\alpha, \beta]$ cannot have more than $n-1+\frac{n}{\ln 2} C|\beta-\alpha|$ isolated zeros.

An analog of this result for a system of ordinary differential equations, viewed as a vector field in space, would concern the number of isolated intersections between integral trajectories of the vector field and hyperplanes (or, more generally, hypersurfaces). For polynomial systems of degree $d$ on $\mathbb{R}^{n}$ of the form

$$
\dot{x}_{i}=v_{i}(t, x), \quad i=1, \ldots, n, \quad v_{i}(t, x)=\sum_{k+|\alpha| \leq d} v_{i k \alpha} t^{k} x^{\alpha},
$$

and algebraic hypersurfaces given by $\{P=0\}$ where $P=P(t, x)$ is a polynomial of degree $d$, the following theorem, proved in [3] (see also [2]), gives a bound for the number of isolated intersections in case the magnitude of the domain of the solution and the amplitude of the solution are controlled by the height of the polynomial system, that is, the number $\max \left\{\left|v_{i k \alpha}\right|: k+|\alpha| \leq d, i=1, \ldots, n\right\}$.

Received by the editors July 31, 2000 and, in revised form, September 11, 2000.

1991 Mathematics Subject Classification. Primary 34C10, 34M10; Secondary 34C07.

Key words and phrases. Bounded oscillation, linear differential equations.

The author is grateful to S. Yakovenko for drawing his attention to this problem and for many stimulating discussions, and to C. Chicone for amelioration of the final text. This research was supported by the Killam grant of Prof. Milman. 
Theorem 2. Suppose that the height of system (2) is bounded by the positive constant $C$. If $\gamma$ is an orbit of the system contained in the box $B_{C}=\left\{(t, x) \in \mathbb{R}^{n+1}\right.$ : $\left.|t|<C,\left|x_{i}\right|<C\right\}$, then the number of isolated intersections of $\gamma$ and $\{P=0\}$ is at most $(2+R)^{B}$ where $B=B(n, d)$ is an explicit elementary function of $d$ and $n$ whose growth rate is smaller than $\exp \exp \exp \exp (4 n \ln d+O(1))$ as $d, n \rightarrow \infty$.

As mentioned in [3], Theorem 2 is nontrivial even for linear systems

$$
\dot{x}=A(t) x, \quad x \in \mathbb{R}^{n}, \quad A(t)=\sum_{k=0}^{d} A_{k} t^{k},
$$

and linear hyperplanes $\left\{\sum_{i=1}^{n} p_{i} x_{i}=0\right\}$. In this case, the box condition reduces to the requirement that $t \in[-C, C]$; the height condition reduces to the uniform boundedness of the norms of the matrix coefficients $A_{k} \in \operatorname{Mat}_{n \times n}(\mathbb{R})$.

Corollary 3. If, for system (3), $\max \left\{\left\|A_{k}\right\|: k=0, \ldots, d\right\}<C$, then there is a uniform bound (expressible as an elementary function of $C$ ) for the number of isolated zeros of every component of every (vector) solution defined on the interval $[-C, C]$.

A comparison of Theorems 1 and 2 suggests the following question: Can the height condition on the polynomial vector field in Theorem 2 be replaced, for instance, by a bound on the norm $\max _{i=1, \ldots, n,(t, x) \in B_{C}}\left|v_{i}(t, x)\right|$; or, in Corollary 3 can it be replaced by a bound on the norm $\max _{t \in[-C, C]}\|A(t)\|$ ? We will show that this is impossible.

The example. For each integer $d$ (no matter how large), there is a linear $2 \times 2$ system (3) of degree $2 d$ with $\max _{t \in[-1,1]}\|A(t)\| \leq 1$ such that a component of one of its solutions in the box $B_{1}$ has $d$ isolated zeros in the interval $[-1,1]$.

Let $t_{1}, \ldots, t_{d}$ be distinct numbers in the interval $[-1,1]$ and let

$$
a(t):=\lambda\left(t-t_{1}\right) \cdots\left(t-t_{d}\right)
$$

where $\lambda$ is a number chosen so small that $|a(t)|+\left|\dot{a}(t)+a^{2}(t)\right|<1$ whenever $t \in[-1,1]$. While the solution $\phi_{1}(t)=\exp \left(\int_{0}^{t} a(s) d s\right)$ of the differential equation $\dot{x}_{1}=a(t) x_{1}$ has no zeroes, its derivative $\phi_{2}=\dot{\phi}_{1}=a(t) \phi_{1}$ has $d$ zeros and also satisfies the equation $\dot{\phi}_{2}=\left(\dot{a}+a^{2}\right) \phi_{1}$. Hence, the supremum over $[-1,1]$ of the coefficient matrix of the degree $2 d$ polynomial linear system

$$
\dot{x}_{1}=a(t) x_{1}, \quad \dot{x}_{2}=\left(\dot{a}(t)+a(t)^{2}\right) x_{1}
$$

is bounded by 1 , and the second component of the solution $t \mapsto\left(\phi_{1}(t), \phi_{2}(t)\right)$ has $d$ isolated zeros in this interval. Moreover, because the system is linear, a constant multiple of this solution is in the box $B_{1}$.

Remark 1. The example shows that the bound stated in Theorem 1 cannot be extended to derivatives of solutions. Also, by choosing $\lambda$ sufficiently small, the coefficients of system (4) can be made uniformly small in every preassigned complex neighborhood of the real segment $[-1,1]$. Hence, the bounds for oscillation with respect to hyperplanes cannot be achieved in the spirit of [1] by imposing bounds for analytic coefficients in the complex domain. 


\section{REFERENCES}

1. Yu. Ilyashenko and S. Yakovenko, Counting real zeros of analytic functions satisfying linear ordinary differential equations, Journal of Differential Equations 126 (1996), no. 1, 87-105. MR 97a:34010

2. D. Novikov and S. Yakovenko, Meandering of trajectories of polynomial vector fields in the affine $n$-space, Publ. Mat. 41 (1997), no. 1, 223-242. MR 98f:58160

3. Ann. Inst. Fourier 49 (1999), no. 2, 563-609. CMP 99:14

4. S. Yakovenko, On functions and curves defined by ordinary differential equations, Proceedings of the Arnoldfest (Ed. by E. Bierstone, B. Khesin, A. Khovanskii, J. Marsden), Fields Institute Communications, 1999, pp. 203-219. CMP 2000:08

Department of Mathematics, Toronto University, Toronto, Ontario, Canada M5S 3G3

E-mail address: dmitry@math.toronto.edu 